\title{
Modeling of photodegradation process to remove the higher concentration of environmental pollution
}

\begin{abstract}
Environmental organic pollutants are mineralized to harmless final-products such $\mathrm{H} 2 \mathrm{O}$ and $\mathrm{CO} 2$ by photocatalytic advanced oxidation processes (AOPs). In photocatalytic-AOPs, an appropriate concentration of $\mathrm{p}$-Cresol was mixed with certain amount of $\mathrm{ZnO}$ in $500 \mathrm{~mL}$ deionized water according to an experimental-design. Then the mixture was irradiated by UV-A lamp at different $\mathrm{pH}$ for $6 \mathrm{~h}$. At specific time intervals, the sampling was carried out to calculate the efficiency of the photodegradation. Therefore, the photodegradation as a system consists of four input variables such irradiation time, $\mathrm{pH}$, amount of $\mathrm{ZnO}$ and p-Cresol's concentration while the only output was the efficiency. In this work, the system was modeled and optimized by semi-empirical response surface methodology. To obtain the empirical responses, the design was performed in laboratory. Then observed responses were fitted with several well-known models by regression process to suggest a provisional model. The suggested model which was validated by several statistical evidence, predicted the desirable condition with higher efficiency. The predicted condition consisted of irradiation time (280 $\mathrm{min}$ ), $\mathrm{pH}$ (7.9), photocatalyst (1.5 g L-1), p-Cresol (95 mg L-1) and efficiency (95\%) which confirmed by further experiments. The closed confirmation results has presented the removal (efficiency $=94.7 \%$ ) of higher $\mathrm{p}$-Cresol concentration $(95 \mathrm{mg} \mathrm{L}-1)$ at shorter irradiation time in comparison with the normal photodegradation efficiency $(97 \%)$ which included irradiation time (300 min), $\mathrm{pH}$ (7.5), photocatalyst amount (1.5 g L-1) and p-Cresol (75 mg L-1). As a conclusion, the modeling which is able to industrial scale up succeeded to remove higher concentration of environmental organic pollutants with ignorable reduction of efficiency.
\end{abstract}

Keyword: AOPs; Environment; Multivariate-modeling; Optimization; Organic-pollutant; Photocatalyst; Photodegradation; RSM; Water treatment; $\mathrm{ZnO}$ 